\title{
Electrical Activity of the Cerebral Cortex during the Low-Frequency Acoustic Stimulation in Women with Different Characteristics of Alpha Rhythm
}

\author{
Alevtyna Morenko Olena Morenko \\ Lesya Ukrainka Eastern European National University, Lutsk, Ukraine
}

\section{Key Words \\ Electrical activity · Individual a-frequency - Cerebral cortex · Low-frequency acoustic stimulation - Power - Coherence . Women}

\begin{abstract}
Background: Human functional capabilities, to a certain extent, depend on an individual's nervous system. The recording of the spontaneous electroencephalogram (EEG) makes it possible to estimate specific features of the nervous system, and in particular the activity of the human brain. Purpose: This study is aimed at investigating the brain in women with a high or a low individual a-frequency (laF), determined in a quiescent state during low-frequency acoustic stimulation. Methods: The study group consisted of 113 right-handed healthy women in the age group 19-21 years. The study group was divided into 2 categories in terms of the average magnitude of laF - groups with high $(\mathrm{n}=59$, laF $\geq 10.25 \mathrm{~Hz}$ ) and low $(\mathrm{n}=54, \mathrm{laF} \leq 10.25 \mathrm{~Hz}$ ) values of laF. The power and coherence of electrical activity of the cerebral cortex as well as the differences between the groups were evaluated in a quiescent state during low-frequency acoustic stimulation. Results: In case of low-frequency acoustic stimulation, a high rate of EEG $\theta-, \beta 2$-power, $\gamma$-waves in the
\end{abstract}

cortex, a1-oscillations - in frontal areas were registered in women with a high a-frequency, while the power a2-, a3and $\beta 1$-activity became lower in the cortex. Women with a low a-frequency had a decrease of the cerebral cortex $\alpha-\theta-$, $a$ - and $\beta$-activity. This was recorded as more generalized, and the expression of $\gamma$-waves was more localized. All the participants had increased values of coherence of the EEG frequency components in the cortex. Some overexertion on the right side was detected in the right hemisphere posterior structures. Some local reduction of the coherence of $\theta-$, a 1-, a3-oscillations was found in women with a high a-frequency and a 1 -activity among the study group members with a low a-frequency in the frontal and anterior temporal areas. Conclusion: The establishment of common factors in the study group is an important step towards defining clear prognostic criteria for the physiological capabilities of people based on the congenital features of brain function.

(c) 2016 S. Karger AG, Basel

\section{Introduction}

In recent times, most professions involve the utilization of modern technologies and technical aids. The latter causes complications in the sphere of mental information

\section{KARGER}

E-Mail karger@karger.com www.karger.com/aon
C 2016 S. Karger AG, Basel

0972-7531/16/0234-0235\$39.50/0
Alevtyna Morenko

Lesya Ukrainka Eastern European National University

13 Voli Avenue, Lutsk 43000 (Ukraine)

E-Mail alevmore@gmail.com 
processing and leads to conditions which are abnormal in the functional sensory capabilities and brain activity of individuals. For this reason, the scientific community is devoting a great deal of attention to the individual characteristics of any brain activity involved in the regulation of human sensory perception. Earlier studies [1-3] show that the cortical potentials depend on the complexity of the stimuli, gender and age of participants, and on their individual experience in responding to any auditory seizures (signals and language). It is evident that sensory perception causes significant changes in the activity of the cerebral cortex; however, precise findings related to cortical changes have so far been quite limited. It cannot be ruled out that certain indicators of cerebral functioning exist which can demonstrate the quality-related characteristics of sensory perception.

It is believed that the pattern of spontaneous electrical activity in the cerebral cortex of one individual or another is genetically determined by features of the structural and functional organization of the human brain [4-6]. Any individual variability in the amplitude-frequency characteristics of alpha $(\alpha)$ rhythms of the electroencephalogram (EEG), in particular the modal frequency of a-rhythms [7-11], has the greatest information among the different rhythms of the background EEG in determining the state of key psychophysiological functions of an individual. Any prevalence in the background EEG in comparison with the low or high $a$-rhythms is described as a prognostic index of the effectiveness of different kinds of professional activity and intellectual abilities, according to Bazanova and Aftanas [7], Umriukhin et al. [12], Klimesch et al. [13], Angelakis et al. [14], Kaplan and Borisov [15], and Rusalova [9]. Their studies are based on the fact that modal $\alpha$-rhythms EEG frequency are strictly determined by genetic trait [16-18], as it reflects important inherited pathways of the structural organization of thalamic and cortical neurons including features of ion processes in those cells $[19,20]$.

Upon consideration of data in existing literature [7, 9, 12-20], we can assume that the modal frequency of the human EEG $a$-rhythm can be determined in quiescent intervals and may have a relation to features of cerebral processes during the process of human sensory perception. In an attempt to deal with this problem, we demonstrated the relationship between the ranges of the male modal alpha frequency and peculiarities of changes in the power and coherence of frequency components of the EEG at a rhythmic low acoustic stimulation [21] in our preliminary study. On the basis of the results obtained, all the men with a high modal $\alpha$-frequency at rhythmical acoustic stimulation were characterized by an increase of some low- and high-frequency EEG rhythms and their capacity in the frontal areas and by a decrease in the capacity of $\alpha$ - and $\beta$-oscillations in the posterior temporal, parietal and occipital areas of the cortex. Men with a low modal $\alpha$-frequency are characterized by a generalized decrease in the capacity of $\alpha$ - and $\beta$-oscillations in the cortex. The coherence of EEG frequency components in the terms indicated herein was increased in all the participants. Considering the results obtained in the male group, it is imperative to determine the significant value of the biological factor of the person's sex, leading to the creation of a single view regarding the relationship between the EEG modal $\alpha$-frequency rates (determined in quiescent intervals) and brain processes during the course of human sensory perception.

The purpose of this study is to shed light on the issue relating to changes in the brain under low-frequency acoustic stimulation in women with a high and low output individual $\alpha$-frequency ( $\mathrm{I} \alpha \mathrm{F}$ ) of the EEG.

\section{Methods}

\section{Objective of the Study}

The study group consisted of 136 female volunteers in the age group 19-21 years, each of whom had given their written consent. Biomedical ethics rules, in accordance with the Helsinki Declaration of the World Medical Association on Ethical Principles of Scientific and Medical Research involving human subjects, were adhered to during the experiment. All the participants were healthy and had normal hearing, based on the judgment and advisory conclusions of medical professionals. The survey of women was conducted during the secretory phase of their menstrual cycle.

\section{Psychophysiological Examination}

As part of the psychophysiological testing, the profile of manual and auditory asymmetry was determined for each subject by the nature of responses in the survey, the execution of the motor and psychoacoustic tests, and the calculation of the individual ratio of manual and auditory asymmetries $\left(\mathrm{K}_{\text {skew }}\right)$ (formula 1$)$ [22].

$$
\mathrm{K}_{\text {skew }}=\frac{\Sigma_{\text {right }}-\Sigma_{\text {left }}}{\Sigma_{\text {right }}+\Sigma_{\text {left }}} \times 100 \%
$$

where $\Sigma_{\text {right }}$ is the number of tasks in which a right hand (or right ear) is dominant and $\Sigma_{\text {left }}$ is the number of tasks in which the left hand (or left ear) is dominant.

Further studies involved dextral participants whose coefficients of manual and auditory asymmetries were positive and above 50\%. The total number of women involved was 113 . 
The effectiveness of the sensory perception was taken as the measurement criteria when the time required for simple and complex sensory and motor reactions was evaluated (the computer system 'Diahnost - 1' was used).

All the examinations were carried out in the morning. The profile of the asymmetry and the time needed for simple and complicated sensory-motor reactions was evaluated $30 \mathrm{~min}$ before the EEG recording registration. Thus, this had no influence on the experiment, particularly on EEG results.

\section{EEG Testing Procedures}

The participants were in a quiescent state with their eyes closed and in a reclining position with their limbs relaxed and not crossed during the EEG testing. The experiment was carried out in a room which was sound- and light-proof. The experimental procedure included the following steps for each participant:

Step 1. The EEG was recorded in a state of functional rest (background);

Step 2. The EEG was recorded with low-frequency acoustic stimulation.

Each step lasted $40 \mathrm{~s}$. To exclude any peripheral effects, the EEG recording registration was started $15 \mathrm{~s}$ after the beginning and stopped at $5 \mathrm{~s}$ on completion.

An electronic version of the drum battle (the software of Finale 2006) was used as the low-frequency acoustic stimulation. Binaural stimuli were produced by 4 speakers placed in different corners of the room at a distance of $1.2 \mathrm{~m}$ from the participant's right or left ear. The stimulus duration was $130 \mathrm{~ms}$; the playback sound volume did not exceed 55-60 dB from the speakers under the measurements carried out by the sound level meter of the ' $\mathrm{DE}$ 3301' type (certificate of attestation \# 025-2009, valid until December 21, 2014). Additionally, the loudness was individually regulated for each participant to achieve the desired level. The rate of sound stimuli delivery was $2 \mathrm{c}^{-1}$.

Registration and Primary Analysis of EEG Data

Active electrodes were placed in accordance with the international system $10 / 20$ in 19 points on the scalp of the head during the EEG ('Neurocom', and the Certificate of State registration \# 6038/2007, valid until April 18, 2014) recording. The performance of the EEG recording was monopolar, with the use of ear electrodes as a reference. The Fourier analysis period was $4 \mathrm{~s}$ with a $50 \%$ overlap. The duration of the sample was $40 \mathrm{~s}$. ICA-procedure analysis was used for the rejection of EEG anomalies.

Both the power $\left(\mu \mathrm{V}^{2}\right)$ and the coherence of the brain electrical activity in the $\theta-, \alpha-, \beta-$, and $\gamma$-frequency intervals were evaluated. Taking into consideration the functional heterogeneity of different sub-bands of the EEG $\alpha$ - and $\beta$-rhythms, the changes in the power and coherence of each of them were considered, and coefficients of coherence above 0.5 analyzed.

The mode of the EEG $\alpha$-rhythm spectral power was determined for each participant at each EEG lead and when the participants were motionless and had their eyes closed. Its value was averaged for all the leads; value obtained was considered as I $\mathrm{F}$ F for each participant $(\mathrm{IaF}, \mathrm{Hz})[13,14]$. The average value of the index was calculated for all women.

Conditional distribution of the sample was accepted. The participants who had a value of I $\alpha \mathrm{F}$ less than average belonged to the study group with a low IaF. The participant with a value of IaF higher than average, were included in the study group with a high IaF.

Activity of the Cerebral Cortex during the

Low-Frequency Acoustic Stimulation
The EEG frequency interval limits were determined individually, relying on the value of the participant's IaF. EEG $[13,14]$ was used and its accuracy was such that the upper limit of a3-subband was set to the right side of the I $\alpha \mathrm{F}$ in increments of $2 \mathrm{~Hz}$. It corresponded to the lower limit of the $\beta 1$-band. The upper limit of the $\beta 1$-subband was defined according to the standards as $25 \mathrm{~Hz}$. The lower limit of the a2-band was determined in steps of $2 \mathrm{~Hz}$ to the left of the peak, and the a1-band in $4-\mathrm{Hz}$ steps, as well as $\theta$-frequencies - in $6 \mathrm{~Hz}$. Limits of $\beta 2$ - and $\gamma$-bands were recognized as standards, $26-35$ and $36-45 \mathrm{~Hz}$.

The resulting individual values of the power and coherence of EEG oscillations within the selected groups of women were averaged for each lead.

\section{Statistical Analyses}

A statistical data analysis was performed using the package 'STATISTICA 6.0' (Stat-Soft, 2001). Any normalcy of the data distribution in the participants' subgroups was evaluated by means of the Shapiro-Wilks test. Based on the test results, it was found that all the studied samples had a normal data distribution. To estimate the significance of differences existing in participants' subgroups, the Student's t test (indicator $\mathrm{t}$ ) was used between steps of testing for both independent and dependent samples. Significant differences between participants' subgroups and among steps of testing were statistically considered at $\mathrm{p} \leq 0.05$ and $\mathrm{p} \leq 0.01$.

\section{Results}

The Individual Modal Frequency Evaluation of the $\alpha$-EEG Activity and Individual Limits of the Frequency Content of the EEG Sub-Range in Female

Participant's Findings

The average value of the modal frequency of any a-activity in samples of female participant was $10.25 \pm$ $0.03 \mathrm{~Hz}$. Considering the leveled nature of the $\mathrm{I} \alpha \mathrm{F}$ value histogram (fig. 1), it was made the conditional distribution of samples under the average mean of the modal frequency of $\alpha$-activity. Two groups were formed, in particular, groups having a high value of $\mathrm{I} \alpha \mathrm{F}(\mathrm{n}=59, \mathrm{I} \alpha \mathrm{F}$ $\geq 10.25 \mathrm{~Hz}$ ) and groups having a low value of $\mathrm{I} \alpha \mathrm{F}(\mathrm{n}=54$, $\mathrm{IaF}<10.25 \mathrm{~Hz}$ ).

\section{Psychophysiological Examination}

Women with a high $\mathrm{I} \alpha \mathrm{F}$ had a shorter time for simple and complex reactions (table 1). This is evidenced by better speed abilities of nervous processes during the time of the rhythmical acoustic stimulation in comparison to those having a low IaF.

\section{Changes in Power and Coherence during the Acoustic Stimulation in the Participant's Groups}

The spectral analysis of the EEG oscillation power during the rhythmic acoustic stimulation in women with a 
Fig. 1. Histogram of values of $\alpha$-frequency mode in female participants. Vertical columns - individual values of the EEG $a$-frequency mode in samples involving female participants.
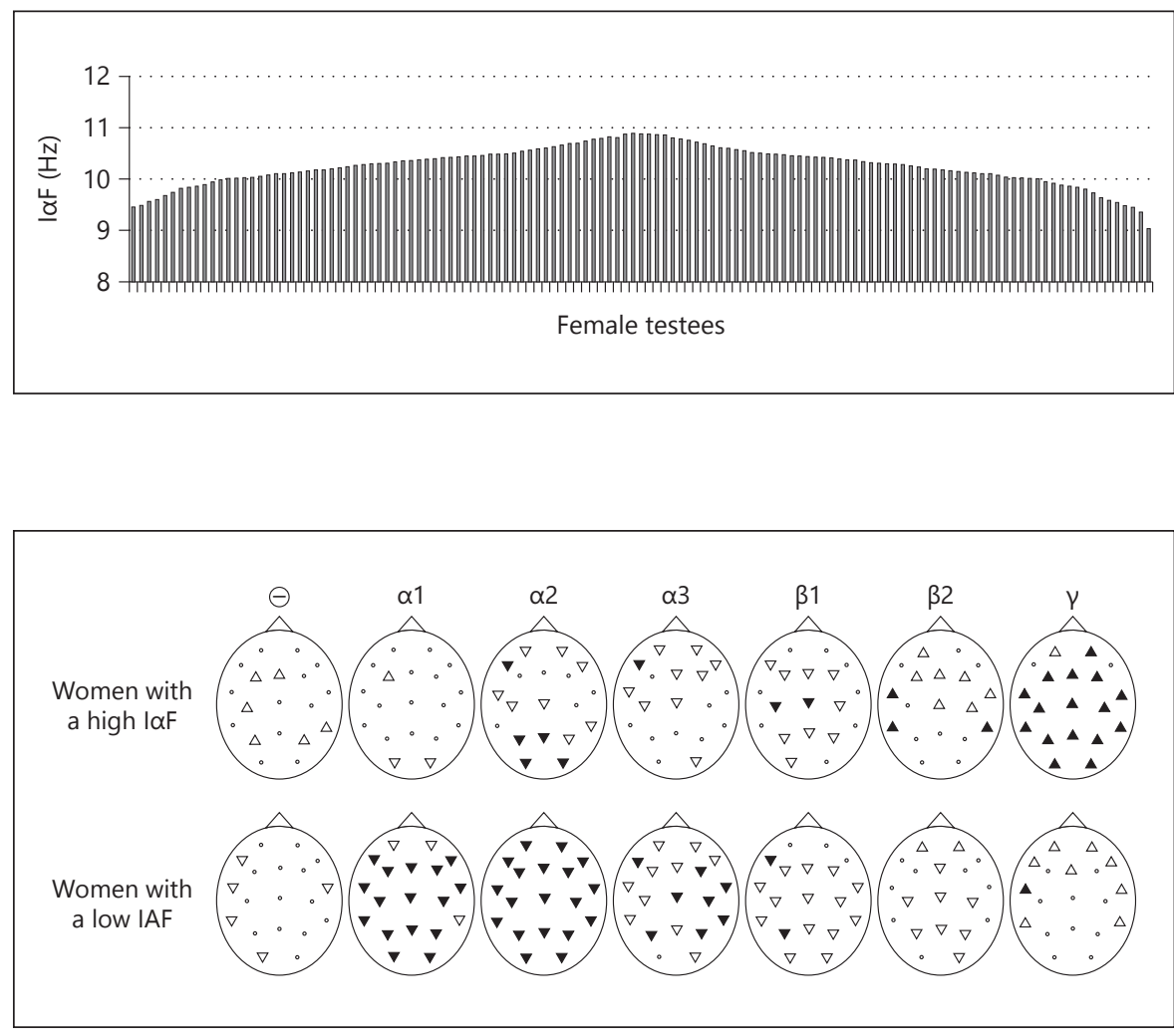

Fig. 2. Topo maps of changes in EEG power fluctuations during the low-frequency acoustic stimulation by female subgroups. Increase (triangle up)/decrease (triangle down) of EEG power fluctuations during the low-frequency acoustic stimulation compared to power in a quiescent state, $\mathrm{p} \leq$ 0.05 (white triangle), $\mathrm{p} \leq 0.01$ (black triangle).
Table 1. Results of psycho-physiological tests characterizing time of simple and complex sensors and motor reactions in women

\begin{tabular}{lll}
\hline Group value & \multicolumn{2}{l}{ Women } \\
\cline { 2 - 3 } & with high IaF & with low IaF \\
\hline Simple reaction time, ms & $253.40 \pm 6.54$ & $322.52 \pm 6.6^{*}$ \\
Selection reaction time, ms & $378.33 \pm 8.77$ & $435.74 \pm 9.4^{*}$ \\
\hline
\end{tabular}

* Indicators of significant differences between the groups of participants with a high and low IaF, $\mathrm{p} \leq 0.05$ to $\mathrm{p} \leq 0.01$.

high $\mathrm{I} \alpha \mathrm{F}$ revealed that $\alpha 2-, \alpha 3-, \beta 1-\mathrm{EEG}$ oscillation power began to decrease in the cortex while $\alpha 1$ activity decreased only in the occipital areas ( $\mathrm{p} \leq 0.05, \mathrm{p} \leq 0.01$; fig. 2 ). This could be a reflection of processes of the active sensory information processing, namely, sensory and spatial analysis of stimuli, their identification and a great attention to the external afferent activity [22-24]. Whereas, a higher generalization and significance of the electrical activity depression in the range of $\theta-, \alpha$ - and $\beta$-EEG of the cortex was found in women with a low $\mathrm{I} \alpha \mathrm{F}$, and this may be the evidence of the increasing role of non-specific activation processes.

At the same time, women with a high IaF have an increase in the $\theta$-, $\alpha 1$ - (in the frontal area), $\beta 2$ - (in the frontal and temporal areas) power of EEG components. Some increase in $\theta$-power and a 1 -activity was observed in the frontal area studied, and in accordance with the views of Machynska [25], Morenko [21], Klimesch et al. [13] may correlate with the selective modulation of the cortical activity areas involved in the information processing and facilitating of the flow of necessary operations. The expression of $\beta 2$-rhythm in the frontal and temporal areas of the cortex in women with a high IaF might be interpreted as the electrophysiological index of the 'differentiated attention' increase [26].

All the tests prove that power increases in the $\gamma$-activity in the participants' cortex ( $\mathrm{p} \leq 0.01$; fig. 2 ) is more significant and generalized in the study group with a high $\mathrm{I} \alpha \mathrm{F}$ that according to Böttger et al. [27], is an activation indicator of a lot of neural networks and strengthens the interaction between spatially distant populations of neurons in the cortex. This leads to the conditions for binding of different sensory, cognitive and executive processes and provides a higher state of readiness and 
Fig. 3. Topo maps of changes of EEG coherence oscillations during the low-frequency acoustic stimulation by female groups. Increase (a solid line)/decrease (a dotted line) of EEG coherence oscillations during the low-frequency acoustic stimulation compared to the coherence in a quiescent state, $\mathrm{p} \leq 0.05$ (a thin line), $\mathrm{p} \leq 0.01$ (a thick line).

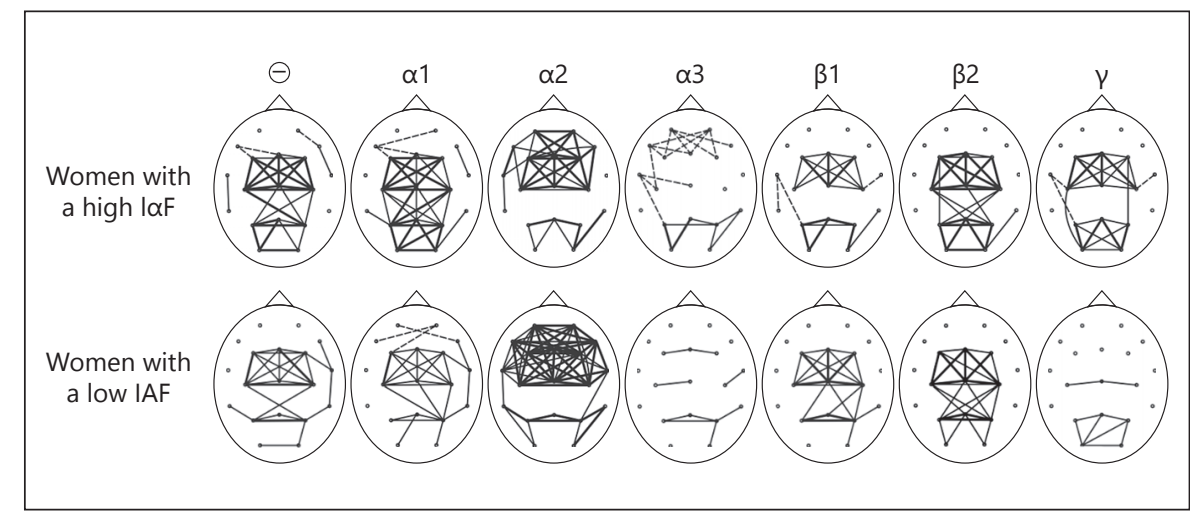

focus (standby) of sensory information [27]. Such a pattern of $\gamma$-waves is associated with a thalamic brain system where neurons were found to have pacemaker properties capable of generating $\gamma$-oscillations and transforming signals to cortical neurons [28]. The beginning of the diffuse $\gamma$-activity is associated with the need to maintain a high level of attention at the time of any activity [29]. According to this data, relatively higher levels of voluntary attention were demonstrated during the acoustic stimulation in the study group, with a high $\mathrm{IaF}$ that correlates with the diffuse character of any $\gamma$-activity. Women with a low I $\alpha \mathrm{F}$ during the acoustic stimulation are characterized by some power increase of $\gamma$-rate whose local character was more in the frontal and temporal areas.

The examination of the coherence relations in the frequency content of the EEG showed an increase in the cortex of all participants ( $\mathrm{p} \leq 0.05, \mathrm{p} \leq 0.01$; fig. 3 ). According to the literature data, the increase of the spatial synchronization of EEG oscillations in the cortex was determined by us subject to these provisions, and had favorable conditions for the excitation spreading in the cortex during sensory information processing $[30,31]$. Changes were greater in the temporal, parietal, and occipital areas of the right hemisphere which probably constitute a corroboration of elements used in the holistic analysis of the participants' sensor stimulation.

A local decrease in the coherence of $\theta$-, $\alpha 1$ - and $\alpha 3$ frequencies was registered along with changes mentioned above in participants with high $\mathrm{I} \alpha \mathrm{F}(\mathrm{p} \leq 0.05)$, and $\alpha 1$-activity in participants with a low $\operatorname{I\alpha F}(\mathrm{p} \leq 0.05)$ in their frontal and anterior temporal areas, particularly, in the left hemisphere. According to the results of the study of a visual attention made by Posner et al. [32], the local desynchronization in the frontal cortex area influences other cortical areas during their direct activities.
This pattern prevailed in women with a high IaF, while coefficients of coherence in the band $\beta$-and $\gamma$-frequencies dominated in women with a low $\mathrm{I} \alpha \mathrm{F}$ that all may indicate a higher nonspecific cortex activity [21, 22, 24] (fig. 3).

\section{Conclusion}

In the case of low-frequency acoustic stimulation, a high rate of EEG $\theta$-, $\beta 2$-power, $\gamma$-waves in the cortex, $\alpha 1$ oscillations - in frontal areas were registered in women with a high $\alpha$-frequency while the power $\alpha 2-$, $\alpha 3$ - and $\beta 1$ activity became lower in the cortex. Women with a low $\alpha$-frequency had a depression of the cerebral cortex $\alpha-, \theta-$, $\alpha$ - and $\beta$-activity recorded as more generalized, and the expression of $\gamma$-waves was more localized. All the participants had an increase in the values of coherence of the EEG frequency components in the cortex. Some overexertion on the right side was detected in the right hemisphere posterior structures. Some local reduction of the coherence of $\theta-, \alpha 1-, \alpha 3$-oscillations was found in women with a high $\alpha$-frequency and $\alpha 1$-activity in participants with a low $\alpha$-frequency in the frontal and anterior temporal areas.

\section{Acknowledgement}

This research was performed under the research project using the state budget funds of Ukraine register No. 0111U002143.

\section{Disclosure Statement}

The authors declare no conflict of interest. 


\section{References}

1 Oknina LB, Kuznetsova OA, et al: Temporal features enable dipole sources P 300 acoustic evoked potential in solving problems of varying difficulty. Hum Physiol 2009;35:5-12.

2 Pavlovych OS, Kotyk OA, et al: Cortical arousal strategies in left-handers during the aural perception and manual playback of mono and polyphonic rhythmical patterns. J Life Sci 2012;6:1408-1413.

3 Panyusheva TD: Musical brain: review of national and international research. Asymmetry 2008;2:41-54

4 Kiroj BN, Belova EI: [Mechanisms of formation and the role of oscillatory activity of neuronal populations in the system of the brain]. Zh Vyssh Nerv Deiat Im I P Pavlov 2000;50: 179-191.

5 Begleiter H, Porjesz B: Genetics of human brain oscillations. Int J Psychophysiol 2006; 60:162-171

6 Van Beijsterveldt CE, Van Baal GC: Twin and family studies of the human electroencephalogram: a review and a meta-analysis. Biol Psychol 2002;61:111-138.

7 Bazanova OM, Aftanas LI: [Individual figures EEG alpha activity and non-verbal creativity]. Sechenov 2007;93:14-26.

8 Danko SG, Ivanitsky GA, et al: General and individual differences in EEG frequency spectrums in solving verbal and spatial tasks. Zh Vyssh Nerv Deiat Im I P Pavlov 2013;63:431436.

9 Rusalova MN: The functional asymmetry of the brain: emotion and activation. Adv Physiol Sci 2003;34:93-112.

10 Hooper GS: Comparison of the distributions of classical and adaptively aligned EEG power spectra. Int J Psychophysiol 2005;55:179-189.

11 Smit CM, Wright MJ, et al: Genetic variation of individual alpha frequency (IAF) and alpha power in a large adolescent twin sample. Int J Psychophysiol 2006;61:235-243.
12 Umriukhin EA, Korobejnikova II, et al: Successful execution of tests by students with different spectral characteristics of the $\alpha$-rhythm of the background EEG. Hum Physiol 2009; 35:33-39.

13 Klimesch W, Sauseng P, Hanslmayr S: EEG alpha oscillations: the inhibition-timing hypothesis. Brain Res Rev 2007;53:63-88.

14 Angelakis E, Lubar JF, et al: Peak alpha frequency: an electroencephalographic measure of cognitive preparedness. Clin Neurophysiol 2004;115:887-897.

15 Kaplan AYA, Borisov SV: Dynamics of the segment characteristics of EEG alpha activity in man at rest and in cognitive load. Zh Vyssh Nerv Deiat Im I P Pavlov 2003;53:2232.

16 Page AJ, O’Donnell TA, Blackshaw LA: Inhibition of mechanosensitivity in visceral primary afferents by $\mathrm{GABAB}$ receptors involves calcium and potassium channels. Neuroscience 2006;137:627-636.

17 Spergel DJ: Calcium and small-conductance calcium-activated potassium channels in gonadotropin-releasing hormone neurons before, during, and after puberty. Endocrinology 2007;148:2383-2390.

18 Anokhin AP, Müller V, et al: Genetic influences on dynamic complexity of brain oscillations. Neurosci Lett 2006;397:93-98.

19 Bellone C, Nicoll RA: Rapid bidirectional switching of synaptic NMDA receptors. Neuron 2007;55:779-788.

20 Ng SC, Raveendran P: EEG peak alpha frequency as an indicator for physical fatigue. Medicon 200;16:517-520.

21 Morenko A: Brain processes during the perception of sensory signals in men with high and low output $\alpha$-frequencies. Ann Neurosci 2014;21:144-149.

22 Boldyreva GN, Zhavoronkova LA, et al: fMRI-EEG - study of the brain of a healthy human reactions on the functional load. Hum Physiol 2009;35:20-30.
23 Bastiaansen MC, Van Der Linden M, et al: Theta responses are involved in lexical-semantic retrieval during language processing. J Cogn Neurosci 2005;17:530-541.

24 Dumenko VN: [Functional significance of high-frequency components of brain electrical activity in the processes of gestalt formation]. Zh Vyssh Nerv Deiat Im I P Pavlov 2002;52:539-550.

25 Machynska RI: Neurophysiological mechanisms of voluntary attention (analytical review). Zh Vyssh Nerv Deiat Im I P Pavlov 2003;53:133-150.

26 Petsche H: Approaches to verbal, visual and musical creativity by EEG coherence analysis. Int J Psychophysiol 1996;24:145-159.

27 Böttger D, Herrmann CS, von Cramon DY: Amplitude differences of evoked alpha and gamma oscillations in two different age groups. Int J Psychophysiol 2002;45:245-251.

28 Pedroarena C, Llinás R: Dendritic calcium conductances generate high-frequency oscillation in thalamocortical neurons. Proc Natl Acad Sci U S A 1997;94:724-728.

29 DanilOva MM, Bukova NB, et al: Caused and Induced Gamma Rhythm in Sensory Coding. Medical Physics, Moscow, Moscow State University Named after MV Lomonosov, 2002, pp 97-106.

30 Pulvermuller F, Birbaumer N, et al: High-frequency brain activity: its possible role in attention, perception and language processing. Prog Neurobiol 1997;52:427-445.

31 Jakovenko IA, Cheremushkin EA, et al: Analysis of induced electrical activisation of the cerebral cortex using wavelet transform at different stages of the installation on the emotional expression. Zh Vyssh Nerv Deiat Im I P Pavlov 2010;60:409-418.

32 Posner MI, Sheese BE, et al: Analyzing and shaping human attentional networks. Neural Netw 2006;19:1422-1429. 